\title{
COLLECTIONWISE NORMALITY WITHOUT LARGE CARDINALS
}

\author{
FRANKLIN D. TALL ${ }^{1}$
}

\begin{abstract}
It was previously known to be consistent relative to a strongly compact cardinal that locally compact perfectly normal spaces must be collectionwise normal. We obtain the same result merely by adjoining $\boldsymbol{}_{2}$ random reals to a model of $V=L$.
\end{abstract}

It is easy to prove that locally compact perfectly normal spaces are collectionwise normal if one assumes Fisher's Axiom, since they are first countable and so Nyikos' method $[\mathbf{N}]$ applies. That axiom requires large cardinals but we shall prove the consistency of this result without assuming anything beyond the consistency of ZFC. The independence is well known: the locally compact modification of the bubble space on a set of reals of power $\boldsymbol{\aleph}_{1}$ or a (special) Aronszajn tree suffice under Martin's Axiom plus $2^{\boldsymbol{N}_{0}}>\boldsymbol{\aleph}_{1}$. Our model is the result of adjoining $\boldsymbol{\aleph}_{2}$ random reals to a model of $V=L$; the proof follows easily from several powerful theorems in the literature.

LEMMA 1. Assume $\diamond$ for stationary systems for all regular cardinals $\kappa \geqslant \boldsymbol{\aleph}_{2}$. Assume $2^{\boldsymbol{\aleph}_{0}} \leqslant \boldsymbol{\aleph}_{2}$ and, for all $\lambda \geqslant \boldsymbol{\aleph}_{2}, 2^{\lambda}=\lambda^{+}$. Then

(1) if $X$ is normal and $\boldsymbol{\aleph}_{1}$-collectionwise Hausdorff, any closed discrete set of points of character $\leqslant \boldsymbol{\aleph}_{1}$ is separated;

(2) if $X$ is normal, $\kappa \geqslant \aleph_{2}$ is regular, and $X$ is $\lambda$-collectionwise Hausdorff for each $\lambda<\kappa$, then any closed discrete set of cardinality $\kappa$ whose points have character $\leqslant \kappa$ is separated;

(3) if $X$ is normal, $\kappa$ is singular, and $X$ is $\lambda$-collectionwise Hausdorff for each $\lambda<\kappa$, then any closed discrete set of cardinality $\kappa$ such that the sup of the character of its points is less than $\kappa$ is separated.

Proof. This follows easily from an analysis of Fleissner's proof in [ $\left.\mathbf{F}_{\mathbf{1}}\right]$.

LEMMA 2. (a) Under the same hypotheses as Lemma 1, if $X$ is a locally compact normal space which is collectionwise normal with respect to collections of $\leqslant \boldsymbol{\aleph}_{1}$ compact sets, then $X$ is collectionwise normal with respect to arbitrary collections of compact sets.

Received by the editors March 17, 1981 and, in revised form, August 25, 1981.

1980 Mathematics Subject Classification. Primary 54A35, 54D15; Secondary 03E35.

Key words and phrases. Locally compact, perfectly normal, collectionwise normal, consistency, $V=L$, random reals.

'The author acknowledges support from Grant A-7354 of the Natural Sciences and Engineering Research Council of Canada. 
(b) If every normal space of character $\leqslant \boldsymbol{\aleph}_{1}$ is $\boldsymbol{\aleph}_{1}$-collectionwise Hausdorff, then every locally compact normal space is collectionwise normal with respect to collections of $\leqslant \aleph_{1}$ compact sets.

Proof. Again, this follows easily from an analysis of Watson's proof in [W], in view of Lemma 1.

Lemma 3 (Gruenhage [G]). If $X$ is locally compact, perfectly normal, and collectionwise normal with respect to compact sets, then $X$ is the disjoint sum of subspaces, each of which is the union of $\leqslant \boldsymbol{\aleph}_{1}$ compact sets.

Lemma 4 (CARLSON [C], $\left.\left[\mathbf{F}_{2}\right]\right)$. Adjoin $\boldsymbol{\aleph}_{2}$ random reals to a model of $\mathrm{CH}$. Then the product measure on $\{0,1\}^{\kappa_{1}}$ can be extended by any $\boldsymbol{\kappa}_{1}$ sets.

We can now prove

THEOREM. Adjoin $\aleph_{2}$ random reals to a model of $V=L$. Then every locally compact perfectly normal space is collectionwise normal.

Proof. By Lemma 4 and Nyikos' method [N], normal spaces of character $\leqslant \boldsymbol{\aleph}_{1}$ are $\boldsymbol{\aleph}_{1}$-collectionwise Hausdorff. Since $\diamond$ for stationary systems holds for regular $\kappa \geqslant \boldsymbol{\aleph}_{2}$ in $L[A], A \subseteq \boldsymbol{\aleph}_{2}$, by Lemmas 1 and 2 we therefore have locally compact perfectly normal spaces are collectionwise normal with respect to compact sets since their character is $\leqslant \boldsymbol{N}_{1}$. Thus Lemma 3 applies, so to get collectionwise normality in general, it suffices to consider a locally compact perfectly normal space $X=\cup_{\alpha<\omega_{1}} F_{\alpha}, F_{\alpha}$ compact, and hence discrete collections of closed sets $K=$ $\cup_{\alpha<\omega_{1}}\left(K \cap F_{\alpha}\right)$. Note that such collections have cardinality at most $\boldsymbol{\kappa}_{1}$, else some $F_{\alpha}$ would admit a large discrete collection, contradicting compactness. Further note that each $K \cap F_{\alpha}$ as a set has countable character in $X$, since it is a compact subset of a locally compact perfectly normal space. Again apply Nyikos and Carlson, treating the $K \cap F_{\alpha}$ 's as points, to complete the proof. More precisely, since the $K \cap F_{\alpha}$ 's have character $\leqslant \boldsymbol{\aleph}_{1}$ and there are only $\boldsymbol{\aleph}_{1}$ of them, there are only $\boldsymbol{\aleph}_{1}$ sets by which the measure on $\{0,1\}^{\boldsymbol{N}_{1}}$ has to be extended. (Note that under merely $V=L$ there is no reason to believe the $K$ 's can be separated from each other, since we have no control over their character as points.)

REMARK. We do not know if our results follow from $V=L$ or are consistent with $\mathrm{CH}$. Their denial is consistent with GCH by Devlin-Shelah [DS]. The question of whether "perfectly" can be eliminated, even with large cardinals, seems to be difficult and important. There are some straightforward generalizations of our theorem, for example $2^{\aleph_{0}}$ can be anything reasonable, but at present these do not seem worth stating.

\section{REFERENCES}

[C] T. Carlson, Removing the Laver ideal from "Extending Lebesgue measure by countably many sets", handwritten manuscript, 1980.

[DS] K. J. Devlin and S. Shelah, A note on the normal Moore space conjecture, Canad. J. Math. 31 (1979), 241-251. 
[F] W. G. Fleissner, Normal Moore spaces in the constructible universe, Proc. Amer. Math. Soc. 46 (1974), 294-298.

$\left[\mathbf{F}_{2}\right] \ldots$, Handbook of set-theoretic topology, North-Holland, Amsterdam (to appear).

[G] G. Gruenhage, Paracompactness and subparacompactness in perfectly normal locally compact spaces, Uspehi Mat. Nauk 35 (1980), 44-49 = Russian Math. Surveys 35 (1980), 49-55.

[N] P. J. Nyikos, A provisional solution to the normal Moore space problem, Proc. Amer. Math. Soc. 78 (1980), 429-435.

[W] W. S. Watson, Locally compact normal spaces in the constructible universe, Canad. J. Math. (to appear).

Department of Mathematics, University of Toronto, Toronto, Ontario M5S 1Al, Canada 